\title{
Monitoring the penetration and accumulation of gold nanoparticles in rat skin ex vivo using surface-enhanced Raman scattering spectroscopy
}

\author{
Honglian Xiong*, ${ }^{*}$, Zhouyi Guo ${ }^{\dagger}$, Huiqing Zhong ${ }^{\dagger}$ and Yanhong $\mathrm{Ji}^{\star}, \S$ \\ *Sun-Yat sen University Cancer Center \\ Guangzhou 510060, P.R. China \\ ${ }^{\dagger}$ Institute of Laser Life Science, College of Biophotonics \\ South China Normal University, Guangzhou 510631, P.R. China \\ *Guangdong Provincial Key Laboratory \\ of Quantum Engineering and Quantum Materials \\ School of Physics and Telecommunication Engineering \\ South China Normal University \\ Guangzhou 510006, P.R. China \\ \$iigh@scnu.edu.cn
}

Received 19 October 2015

Accepted 27 December 2015

Published 1 March 2016

\begin{abstract}
Contamination by accidental cutaneous contact with the commercial products and the air pollutants raised a considerable health and safety issue. This study aimed to trace the dynamics of the $20 \mathrm{~nm}$ gold nanoparticle (GNP) penetration and accumulation in rat skin tissues using a surface-enhanced Raman scattering (SERS) technique. After the topical application of GNPs on rat skin surface, the SERS spectra were recorded for every $15 \mu \mathrm{m}$ to an overall depth of $75 \mu \mathrm{m}$ from skin surface for $150 \mathrm{~min}$. The processes of GNP penetration in rat skin were accompanied by aggregation of GNPs, which affected SERS spectra. The results revealed that $20 \mathrm{~nm}$ GNPs can penetrate through stratum corneum layer, viable epidermis layer, and then into dermis layer. This study demonstrated for the first time the potential of SERS spectroscopy to monitor the penetration and accumulation of GNPs in rat skin.
\end{abstract}

Keywords: Surface-enhanced Raman scattering spectroscopy; rat skin; gold nanoparticle penetration; aggregation.

\section{Introduction}

Particle matter $(\mathrm{PM})$ is one of the ambient air particulate pollutants, which can be classified into three groups based on their diameters: $<0.1,0.1-2.5$, and $>2.5 \mu \mathrm{m}$. PM2.5 in ambient air can easily transfer into human bodies by inhalation, ingestion, dermal

$\S_{\text {Corresponding author. }}$

This is an Open Access article published by World Scientific Publishing Company. It is distributed under the terms of the Creative Commons Attribution 4.0 (CC-BY) License. Further distribution of this work is permitted, provided the original work is properly cited. 
contact absorption, deposit in many organs, and pose a hazard to human health. "Nanoparticles" are engineered structures with diameters of $<100 \mathrm{~nm}$. Due to smaller size, big surface area, and suspension in air for longer time, nanoparticles show greater toxicity than fine particulates $(<2.5 \mathrm{um})$ of the same material on a mass basis. ${ }^{1}$ Recently, with the development of nanotechnology, nanomaterials are widely investigated and applied in many fields, such as biomedicine, cosmetics, catalysis, food, textiles, semiconductors, and biomedicines. ${ }^{2-5}$ In addition to occupational exposure, direct human bodies exposures through commercial applications and ambient air pollution were a major concern.

Gold nanoparticles (GNPs) are one of the most extensively studied nanomaterials first described by Faraday more than 100 years ago. ${ }^{6}$ Due to its easy preparation, functionalization, size and shape control, and good biocompatibility, GNPs have become a hot issue in the fields of drug delivery, bioimaging, biosensors, and catalysis. ${ }^{7-9}$ Meanwhile, the risks of contamination with nanomaterials by accidental cutaneous contact with the commercial products and the air pollutants had received considerable attention. The toxicity of nanomaterials is mainly due to its biological permeability. Humans contact with the different air pollutants is primarily via four exposure route: the respiratory tract (i.e., inhalation exposure), skin, eyes, and the gastrointestinal tract. The human skin was the largest organ with a surface area of nearly $1.5 \mathrm{~m}^{2}$ in the body, protecting us from the unwanted environmental effects. The skin's most important function was to form an effective barrier between the organism and the environment preventing the invasion of pathogens and fending off chemical and physical assaults, as well as the unregulated loss of water and solutes. Polar and nonpolar materials can permeate across the stratum corneum (SC) via a paracellular route. The potential of nanoparticles to penetrate into the SC and to diffuse into underlying structures raised a considerable health and safety issue. Hence, understanding their penetration abilities into the skin was necessary. In recent years, skin penetration and accumulation of metal nanoparticles had been investigated through several techniques, including scanning electron microscopy (SEM), Two-photon microscopy combined with fluorescence lifetime imaging microscopy (FLIM) analysis, ${ }^{10}$ confocal Raman microscopy, ${ }^{11}$ transmission electron microscope (TEM), static Franz cells, reflectance confocal microscopy (RCM), multi-photon tomography (MPT), and optical coherence tomography (OCT). ${ }^{12-16}$ Although these modalities were useful, they presented certain limitations. SEM, TEM, RCM, MPT, and Franz cells had an excellent resolution and can be used to detect nanoparticle penetration in skin. However, these methods were destructive and difficult for practical implementation. OCT was a suitable tool for noninvasive imaging of the process of nanoparticle penetration and accumulation in skin by determining changes in optical characteristics of the OCT images. These changes can indicate the penetration and accumulation of nanoparticles in tissues. However, OCT did not provide a high resolution. Darvin et al. used Ag NPs $(70 \pm 20 \mathrm{~nm})$ to investigate human skin penetration ex vivo and verified the location of the penetrated AgNPs using SERS technology. Their conclusions showed that the coated AgNPs were able to penetrate the SC of the skin. Confocal Raman microscopy, especially SERS technology, provided high sensitivity and has a much higher log-order signal than normal Raman scatterings.

Compared with these techniques, surface-enhanced Raman scattering (SERS) technology had shown a great promise for monitoring the process of nanoparticle-biotissue interaction by changes in optical characteristics in real time and noninvasively. SERS effect, an enhancement phenomenon of the Raman effect, was a hot issue in the application of GNPs. SERS effect allowed for label-free, highly sensitive and selective detection of low concentration analytes through amplification of localized plasmon resonances of noble metal nanoparticles. The SERS effect resulted in strongly increased Raman signals from molecules which had been attached to nanometer sized metallic structures. Previous reports demonstrated that SERS enhancement levels were mainly associated with electromagnetic (EM) fields. ${ }^{17-19}$ This implied that enhancement factor strongly depended on the morphology of nanoparticles, such as size, shape, and aggregation. In the aggregates, large EM fields were created at the junction sites of adjacent nanoparticles (hot spots). ${ }^{17,20-22}$ So far, extremely high enhancement factors had been obtained by exploiting EM fields on aggregates formed by silver or GNPs with sizes of 20$60 \mathrm{~nm} .{ }^{23}$ Therefore, SERS technology based on metal aggregates can be used to deliver enhanced Raman signals and provided detailed information on their environments. ${ }^{24-26}$ Kneipp et al. monitored the changes in the morphology of GNPs without Raman 
reporter molecules in living cells over time by SERS spectroscopy. ${ }^{27}$ The increase in SERS signals in cells was indicative of the formation of gold nanoparticle aggregates (GNAs). Sharma et al. measured nanoparticle penetration through bone by surfaceenhanced spatially offset Raman spectroscopy. ${ }^{28}$ With this technique, the bone penetration of GNPs coated with a Raman reporter molecule can be detected by specific Raman signature of the nanotags. However, the fate of nanoparticles in tissues had not been investigated. Previous reports had shown that when GNPs penetrate through skin tissues it form aggregates. ${ }^{12,14,15}$ GNPs could hardly generate a SERS enhancement, because an excitation wavelength of $785 \mathrm{~nm}$ used is far away from the surface plasmon absorption band of GNPs, but GNP aggregates produce strong SERS enhancements.

The aim of this study was to continuously monitor the changes in SERS spectra in the rat skin associate with $20 \mathrm{~nm}$ GNPs penetration and aggregation over time at different positions using SERS technology in a noninvasive manner. Reports demonstrated that nanoparticles with the diameter ranging between 7 and $20 \mathrm{~nm}$ could penetrate the skin. Owing to its tunable size, biocompatibility, and easy functionalization, GNPs have been extensively studied on photothermal therapy, SERS, and drug delivery. ${ }^{29}$ SERS spectra were acquired in the wavenumber ranging of $800-1800 \mathrm{~cm}^{-1}$ from rat skin with a $785 \mathrm{~nm}$ excitation laser. These spectra acquired provide information on rat skin and citrate-coated GNAs. By changes in SERS signal, we can conclude about penetration and accumulation of nanoparticles in skin, and trace the dynamics of these processes.

\section{Materials and Methods}

\subsection{Materials}

Chloroauric acid $\left(\mathrm{HAuCl} \cdot 3 \mathrm{H}_{2} \mathrm{O}\right)$ and sodium citrate $\left(\mathrm{Na}_{3} \mathrm{C}_{6} \mathrm{H}_{5} \mathrm{O}_{7}\right)$ were purchased from SigamaAldrich. Milli-Q grade water ((Millipore) was used for all solution preparation and experiments.

\subsection{Preparation of GNPs and GNAs}

The gold colloids in our experiment were synthesized using a modified Frens' method. ${ }^{30}$ Briefly, HAuCl4 solution $(100 \mathrm{~mL}, 0.25 \mathrm{mM})$ was heated until boiling under a reflux condenser. $1 \%(\mathrm{w} / \mathrm{v})$ sodium citrate
$\left(\mathrm{Na}_{3} \mathrm{C}_{6} \mathrm{H}_{5} \mathrm{O}_{7}\right)$ solution $(2.0 \mathrm{~mL})$ was rapidly added into the boiling solution with vigorous stirring. Boiling continued for $10 \mathrm{~min}$, the heating mantle was then removed, and stirring was continued for an additional $15 \mathrm{~min}$. The solution was allowed to cool to room temperature.

For GNAs preparation, $200 \mu \mathrm{L}$ of GNPs were placed in 96 well microtiter plates, and $50 \mu \mathrm{L}$ of the aggregating agent solution (Magnesium sulphate, MgSO4; 0.0167M final concentration) was then added.

\subsection{Characterization of GNPs and GNAs}

The morphology and size of GNPs and GNAs were observed by a SEM. The absorption spectra were characterized in the wavelength range $400-900 \mathrm{~nm}$ using a visible/near infrared (Vis-NIR) spectrometer.

\subsection{Instruments}

Raman spectra and SERS spectra were acquired using confocal Raman microspectrometer (Renishaw InVia, Derbyshire, England) with a semiconductor laser and a Leica DM2500 microscope. Samples were placed on the computer-controlled 3-axis motorized stage. The band of a silicon wafer at $520 \mathrm{~cm}^{-1}$ was used to calibrate spectroscopy. A $20 \times$ microscope objective was used for SERS analysis. The lateral and axial resolution of the instrument was about 1 and 2 $\mu \mathrm{m}$, respectively. The system acquires Raman spectra in the wavenumber range of $800-1800 \mathrm{~cm}^{-1}$ from rat skin with a $785 \mathrm{~nm}$ excitation laser, laser power of $9 \mathrm{~mW}$, exposure time of $4 \mathrm{~s}$ and one-time accumulation. Low laser intensities excludes possible tissue changes caused by laser illumination. Moreover, near-infrared (NIR) excitation is required, because NIR wavelengths can penetrate deeper into tissue while being less absorbed by biological tissue.

\subsection{Skin hematoxylin staining for microscope observation}

Skin specimens exposed to gold colloids for $2.5 \mathrm{~h}$ were frozen, cut into $15 \mu \mathrm{m}$ sections, mounted on poly-L-lysine coated slides, fixed with $4 \%$ formaldehyde for $1 \mathrm{~min}$, stained with hematoxylin, and coverslipped with glycerol gel. ${ }^{14}$ Sections were observed using a light transmission microscope. 


\subsection{Ex vivo penetration study}

Skin penetration experiments were conducted using male Wistar rats weighing about $260 \mathrm{~g}$. All animal experiments were approved by an ethical committee. The abdominal hair was removed from rats with an electric clipper and an electric razor 1 day before the penetration study. ${ }^{16}$ Rats were anesthetized with ether anesthesia and decapitated. The abdominal skin was excised immediately. The tissue samples were sealed for preventing natural dehydration and stored at $4^{\circ} \mathrm{C}$ for no longer than $12 \mathrm{~h}$ before use. The samples were allowed to equilibrate $30 \mathrm{~min}$ at room temperature prior to the experiments. Each skin sample was cut into $2 \times 2 \mathrm{~cm}^{2}$ pieces by microtome before measurement. The penetration experiments were conducted at $22^{\circ} \mathrm{C}$. The underside of the skin was soaked in $0.9 \%$ normal saline to maintain the hydration of the skin, and the epidermis was exposed to air. One droplet of GNPs solution $(\sim 20 \mu \mathrm{L})$ was applied on rat skin. A set of axial scanning from the skin surface to $75 \mu \mathrm{m}$ was taken at the same site after the topical application of GNPs solution. Spectra were acquired for every $15 \mu \mathrm{m}$ to an overall depth of $75 \mu \mathrm{m}$ at various time intervals of 30, 60, 90, and 150 min by Raman microspectroscopy. GNPs solution on the sample was removed right before acquiring the spectrum. Five random sites of each specimen were selected. The maximum and minimum measurements were excluded, and the average of the remaining three measurements was used to comprise the data set.

To accurately evaluate GNP penetration depth, the difference of the refractive index between air and skin was considered, which induces a deviation of the light beam. The correction model developed by Everall is used to correct penetration depth. ${ }^{31,32}$ The real depth, $Z$, is expressed using the following equation:

$$
Z=\Delta\left[m^{2} \frac{N A^{2}\left(n^{2}-1\right)}{\left(1-N A^{2}\right)}+n^{2}\right]^{1 / 2},
$$

where $\Delta$ is the measured optical depth below the skin/ air interface, NA is the numerical aperture of the objective (NA $=0.4), m$ is the pupil factor $(0 \leq m \leq 1)$, and $n$ is the refractive index of the sample. The refractive indexes between different layers of the skin are different. In this study, we selected a middle value of 1.4 as the refractive index of rat tissues.

Equation (1) can be reduced to:

$$
Z=\Delta\left[0.18 m+1.4^{2}\right]^{1 / 2} .
$$

If the term $0.18 m$ is ignored, Eq. (2) is reduced to:

$$
Z=1.4 \Delta \text {. }
$$

\subsection{Data pre-processing}

All of the measured Raman spectra $\left(800-1800 \mathrm{~cm}^{-1}\right)$ were pre-processed. Firstly, a fifth-order polynomial was used for fitting the fluorescence background, and then this polynomial was subtracted from the raw spectrum to yield the tissue SERS spectra. Vancouver Raman algorithm was employed for spectra smoothing and baseline correction.

\section{Results and Discussion \\ 3.1. Characterization of GNPs and GNAs}

SEM was used to study the morphological characteristics of GNPs and GNAs. The average particle size of GNPs was $20 \mathrm{~nm}$ with a good dispersity (Fig. 1(a)). The corresponding GNAs had a variety of sizes and shapes (Fig. 1(b)). GNPs and GNAs were further characterized by a UV-vis spectrometer. The GNPs has one surface plasmon peak which was located at 523 nm (Fig. 1(c)). The prepared GNAs had two bands, which corresponded to a transverse plasmon band $(530 \mathrm{~nm})$ and an EPB (780 nm) (Fig. 1(c)). The results confirmed the aggregation of GNPs with the addition of MgSO4. It was reported that a spherical GNP only exhibit transverse plasmon band, and an elongated particle, such as metal nanoshells, aggregated nanoparticles, and nonspherical nanoparticles, showed a second absorption band. ${ }^{33,34}$ Each GNA with a particular size and shape presented a unique EPB in terms of bandwidth and peak position due to the random nature of aggregate formation. However, in an ensemble averaged solution these bands combined to form one strong, inhomogeneously broadened band. ${ }^{35}$

\subsection{GNP penetration was assessed with specimen histology}

GNP penetration in rat skin tissues was firstly assessed with hematoxylin staining. Skin specimens exposed to gold colloids for $2.5 \mathrm{~h}$ were frozen, cut into $15 \mu \mathrm{m}$ sections, and stained with hematoxylin. ${ }^{14}$ The sections were observed using a light transmission microscope. 


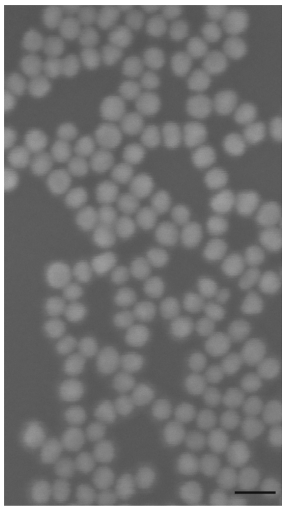

(a)

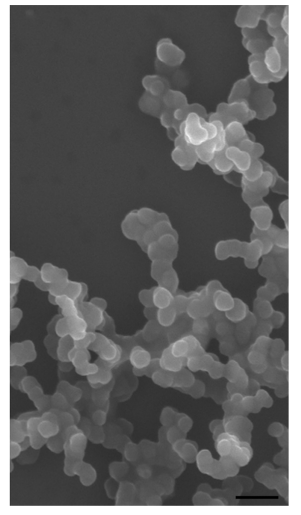

(b)

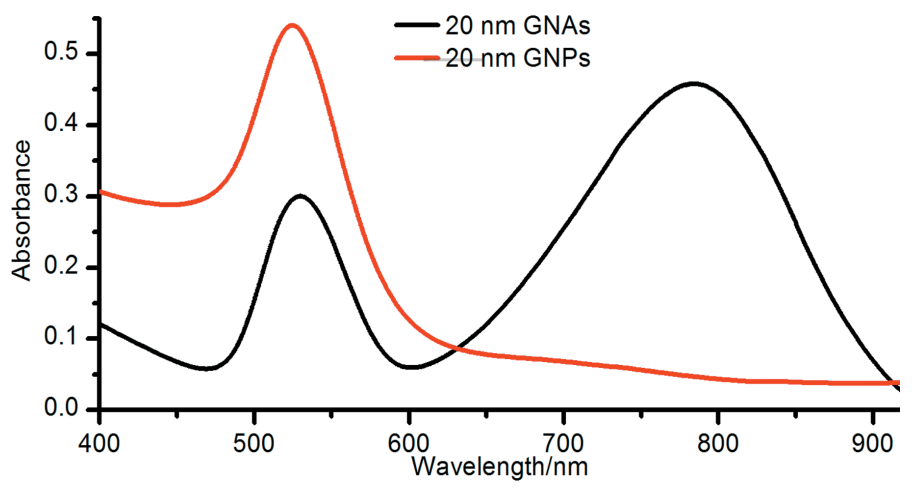

(c)

Fig. 1. SEM images of (a) GNPs with an average particle size of $20 \mathrm{~nm}$ and (b) the corresponding GNAs. Bar $=40 \mathrm{~nm}$. (c) vis-NIR absorption spectra of GNPs and GNAs. The peak absorption of GNPs is located at $523 \mathrm{~nm}$. GNAs have two bands, corresponding to transverse $(530 \mathrm{~nm})$ and extended plasmon bands (EPB) (peaked around $780 \mathrm{~nm}$ ).

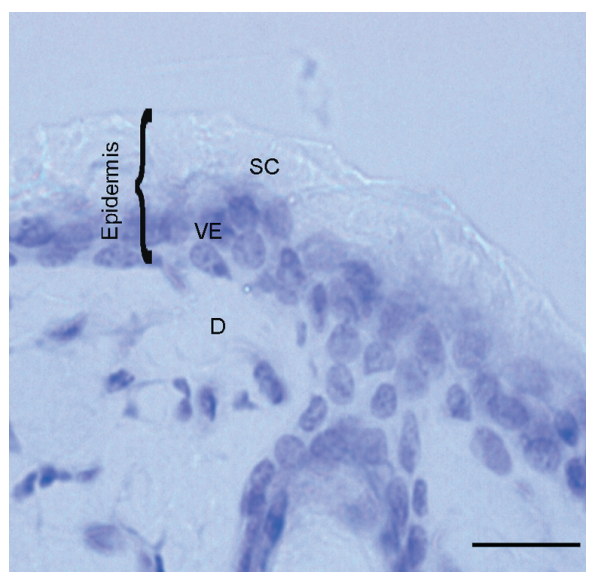

(a)

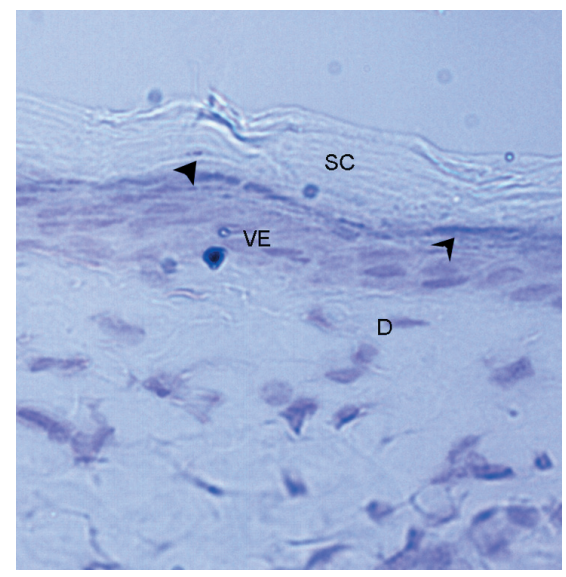

(b)

Fig. 2. Images of hematoxylin-stained rat skin tissues (a) without GNP treatment, and (b) with $20 \mathrm{~nm}$ GNP treatment. Blue deposits were clearly observed in the SC layer and the SC/VE junction (indicated by black arrow) $($ Bar $=50 \mu \mathrm{m}$; magnification $\times 40)$. SC, viable epidermis (VE), and dermis (D).

Skin consists of SC, VE, and D (Fig. 2). No aggregates were presented in skin layers without GNP treatment (Fig. 2). However, blue deposits (indicated by black arrows in Fig. 2(b)) in the SC layer and SC/VE junction of GNP treated rat skin samples were clearly observed.

This finding indicated that GNPs penetrated across SC layer, and then reached and aggregated in the $\mathrm{SC} / \mathrm{VE}$ junction due to skin barrier properties formed by SC and VE layers. It was reported that a more tightly compacted SC layer near VE layer results from an abundance of GNAs. ${ }^{36}$ However, GNAs were not invisible in VE and D layers (Fig. 2(b)). There were two reasons for this. First, GNPs may be washed off from skin layers, in particular, loose VE layer. The sections were repeatedly washed in the process of complicated hematoxylin staining. Second, the low resolution of a light microscope did not enable observation of small GNAs.

\subsection{SERS spectra of citrate-coated GNPs and citrate-coated GNAs}

$\mathrm{MgSO}_{4}$ was added to GNPs to prepare GNP aggregates for assessment of SERS activity of GNPs aggregation. The assessment of the difference of GNPs SERS activity and GNAs was necessary. The SERS spectra of citrate-coated GNPs and GNAs were measured using simple SERS technology. The normal Raman bands of major sodium citrate 


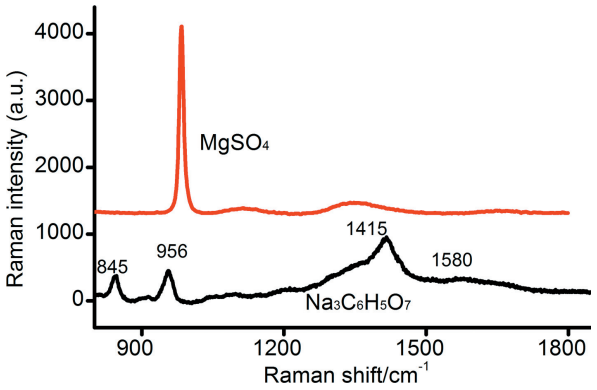

(a)

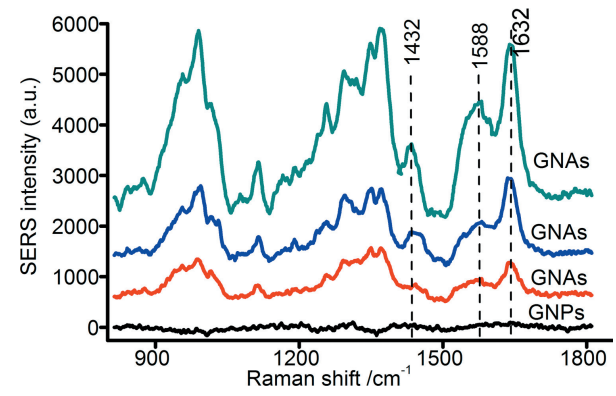

(b)

Fig. 3. (a) Normal Raman spectra of magnesium sulphate $\left(\mathrm{MgSO}_{4}\right)$ and citrate sodium $\left(\mathrm{Na}_{3} \mathrm{C}_{6} \mathrm{H}_{5} \mathrm{O}_{7}\right)$. (b) A SERS spectrum of citrate-coated GNPs and three different spectra of citrate-coated GNAs. No obvious SERS signals for citrate-coated GNPs were visualized. However, intense SERS signals from citrate-coated GNAs were observed. The spectra reflected the information on citrate group in the nanometer-vicinity of GNAs. The SERS signal intensity of each GNA is highly variable, due to the difference of nanoaggregate sizes and shapes in randomly aggregated nanoparticle colloids.

$\left(\mathrm{Na}_{3} \mathrm{C}_{6} \mathrm{H}_{5} \mathrm{O}_{7}\right)$ had been previously measured at $1580,1415,956$, and $845 \mathrm{~cm}^{-1}$, and assigned to $\mathrm{v}_{\mathrm{as}}$ (COO- $), \mathrm{v}_{\mathrm{s}}(\mathrm{COO}-), \mathrm{v}_{\mathrm{s}}(\mathrm{C}-\mathrm{C})$, and $\mathrm{v}_{\mathrm{s}}(\mathrm{CCCC}-\mathrm{O})$ modes, ${ }^{37}$ respectively (Fig. 3(a)). A SERS spectrum of citrate-coated GNPs and three different spectra of citrate-coated GNAs were showed (Fig. 3(b)). No obvious SERS signals for citrate-coated GNPs were visualized, because an excitation wavelength of 785 $\mathrm{nm}$ used in the experiment was far away from the surface plasmon absorption band of the GNPs and the intensity of excitation laser was very low. ${ }^{38,39}$ With the addition of $\mathrm{MgSO}_{4}$ and the formation of GNAs, immediate intense SERS signals at 1432 and $1588 \mathrm{~cm}^{-1}$ from nanoaggregates were detected (Fig. 3(b)). Little enhancement was observed for a single GNP (from 17 to $80 \mathrm{~nm}$ ), but GNP aggregates produced strong SERS enhancements. The enhancements on nanostars or nanoaggregates was several orders of magnitude greater than the SERS signals from nanospheres. ${ }^{40,41}$ The nanoaggregates yielded large enhancements due to the generation of the EM "hot spots" between two nanospheres. And the highest SERS enhancements had been obtained on aggregates or clusters formed by individual silver or GNPs 20-60 nm in size. There was compelling evidence that SERS enhancement levels were associated mainly with enhanced EM fields. ${ }^{42,43}$ The spectra give the detailed information on citrate group in the nanometer-vicinity of GNAs. Moreover, the SERS enhancement levels of three GNAs were very different due to the difference of nanoaggregate sizes and shapes in randomly aggregated nanoparticle colloids. We found that the Raman peaks from citrate-coated GNAs were overlapped with those from sulphate group in the $800-1000 \mathrm{~cm}^{-1}$ region, whereas
SERS bands at 1422 and $1588 \mathrm{~cm}^{-1}$ can be clearly observed (Fig. 3(b)). The peaks of citrate group at 1416 and $1580 \mathrm{~cm}^{-1}$ were shifted to 1422 and 1588 $\mathrm{cm}^{-1}$, respectively. And the peak at $1632 \mathrm{~cm}^{-1}$ was assigned to amorphous carbon created on the gold surface by high intensity laser irradiation. ${ }^{44}$

\subsection{Raman spectra of rat skin at different depth and SERS spectra of the skin after topical application of $\mathrm{GNPs}$}

To study the penetration process of GNPs into rat skin, we first compared the Raman spectra of rat skin samples with those of rat skin treated with GNPs. Normal Raman spectra of rat skin with a 15 $\mu \mathrm{m}$ increment from 15 to $75 \mu \mathrm{m}$ under the skin surface and a SERS spectrum of skin after topical application of GNPs were measured (Fig. 4).

The main Raman bands of skin were observed at the following peak positions: $856 \mathrm{~cm}^{-1}$ (aromatic), $937 \mathrm{~cm}^{-1}$ (stretch backbone), $1004 \mathrm{~cm}^{-1}$ (phenylalanine and urea), ${ }^{45} 1450 \mathrm{~cm}^{-1}$ (proteins and lipids), and $1662 \mathrm{~cm}^{-1}$ (amide I) (Fig. 4(a)). ${ }^{46}$ Although the Raman signals monotonically diminish with increasing depth due to the limited light penetration into rat skin, the Raman peaks were clearly observed at the depth of $400 \mu \mathrm{m}$ below the surface. ${ }^{47}$ We selected two intense bands at 1004 and $1662 \mathrm{~cm}^{-1}$ as the markers for the skin, which are indicated by black arrows (Fig. 4(a)). Moreover, citrate group did not present intense Raman signal in these spectral regions.

A SERS spectrum was collected at $20 \mu \mathrm{m}$ below the skin surface (Fig. 4(b)). The SERS spectra of 


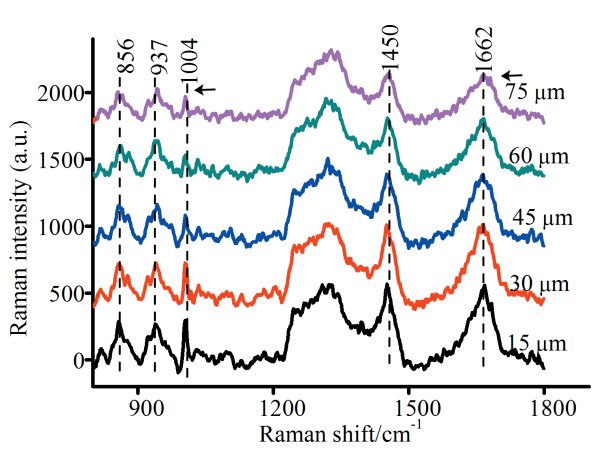

(a)

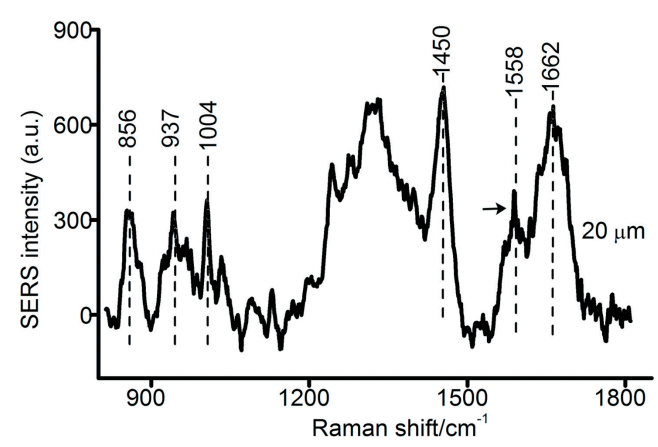

(b)

Fig. 4. (a) Normal Raman spectra of rat skin and (b) SERS spectra of the skin after topical application of GNPs. The SERS spectra of the skin reflected the information on skin and GNAs formed in skin tissues. After topical application of GNPs, as a consequence of their exposure to the changing tissue environment, individual GNPs form nanoaggregates. The peaks at 1004 and $1662 \mathrm{~cm}^{-1}$ were selected as the skin marker. The peak at $1588 \mathrm{~cm}^{-1}$ from the citrate group was used as GNAs marker (arrows indicated in Fig. 4).

the skin give the detailed information on skin and GNAs formed in skin tissues. It contained the spectral features of both skin and citrate-coated GNAs. After the topical application of GNPs, as a consequence of their exposure to the changing tissue environment, individual GNPs form nanoaggregates. The Raman bands of the skin such as 1004, 1450 and $1662 \mathrm{~cm}^{-1}$ can be clearly observed (Fig. 4(b)). For GNAs, we can identify the intense band at 1588 $\mathrm{cm}^{-1}$, which was chosen as the marker indicated by an arrow (Fig. 4(b)).

\subsection{Study of GNP penetration and accumulation in rat skin ex vivo}

To investigate dynamics of $20 \mathrm{~nm}$ GNP penetration, the changes in the SERS spectra was monitored over time at different positions. A series of SERS spectra were acquired by Raman microspectroscopy at the depth of $0,15,30,45,60$, and $75 \mu \mathrm{m}$ after topical application of GNPs for 30,60, 90, and $150 \mathrm{~min}$ (Fig. 5). Five random locations were measured using automated depth scanning at various time intervals. The maximum and minimum measurements were excluded, and the average of the remaining three measurements was used to comprise the data set.

As shown in Fig. 5, these spectra contained the spectral features of skin and citrate-coated GNAs. We found that the skin markers at 1004 and 1662 $\mathrm{cm}^{-1}$ were not significantly enhanced by GNAs. This phenomenon may be because the cellular molecule was difficult in close proximity to SERS hot spots of GNAs in intercellular space. Previous reports demonstrated that analyte molecules must be within 0-4 nm of hot spots for the EM fields to have an effect. ${ }^{33}$ Metal nanoparticles were observed primarily in the intercellular spaces in intact skin. ${ }^{36}$ Additionally, the enhancement factor strongly depended on the sizes and shapes of the GNAs. ${ }^{33}$ Given the random nature of aggregate formation, the GNAs presented a broad distribution. Therefore, the quantitative measurements of nanoparticle concentrations by variations in SERS intensities was difficult.

As shown in Fig. 5(a), after 30 min diffusion, the spectral marker for the GNAs at $1588 \mathrm{~cm}^{-1}$ were only presented at $15 \mu \mathrm{m}(\Delta)$ below the skin surface. After correction by Eq. (3), the real depth $(Z)$ descends from 15 down to $21 \mu \mathrm{m}$ (the location of SC layer, see the images of hematoxylin-stained sections in Fig. 2). These data illustrated that GNAs were presented in the SC layer, but do not reach VE layer. In addition, the Raman signals of tissues were not substantially enhanced, which was likely attributed to weak interaction between tissues and GNAs.

After 60 min diffusion, the GNA marker at 1588 $\mathrm{cm}^{-1}$ appeared at 15 and $30 \mu \mathrm{m}$ (measured optical depth: $\Delta$ ) under the skin surface (Fig. 5(b)). Similarly, after correction, the real depth $(Z)$ descended from 15 and $30 \mu \mathrm{m}$ down to 21 and $42 \mu \mathrm{m}$ ((the location of VE layer (Fig. 2)). The results demonstrated that $20 \mathrm{~nm}$ GNPs can penetrate through the $\mathrm{SC}$ layer and reached the $\mathrm{SC} / \mathrm{VE}$ junction at this time point.

After the topical application of $90 \mathrm{~min}$, the GNA marker at $1588 \mathrm{~cm}^{-1}$ were all detected at $0,15,30$, and $45 \mu \mathrm{m}$ (measured optical depth: $\Delta$ ) under the skin surface (Figs. 5(c) and 5(d)). After correction 


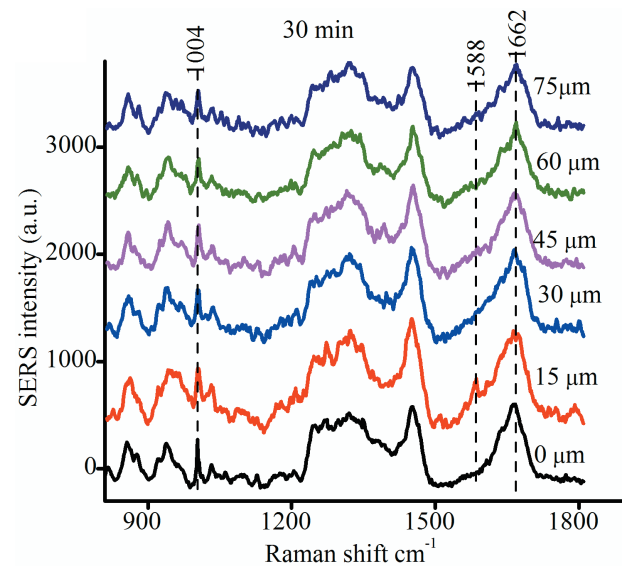

(a)

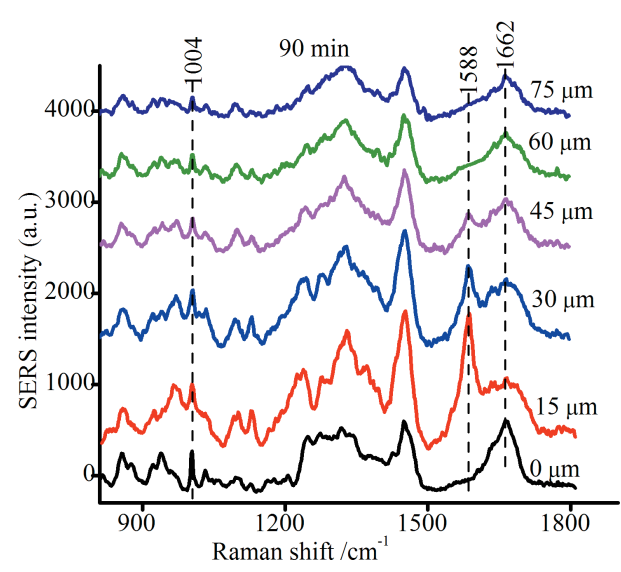

(c)

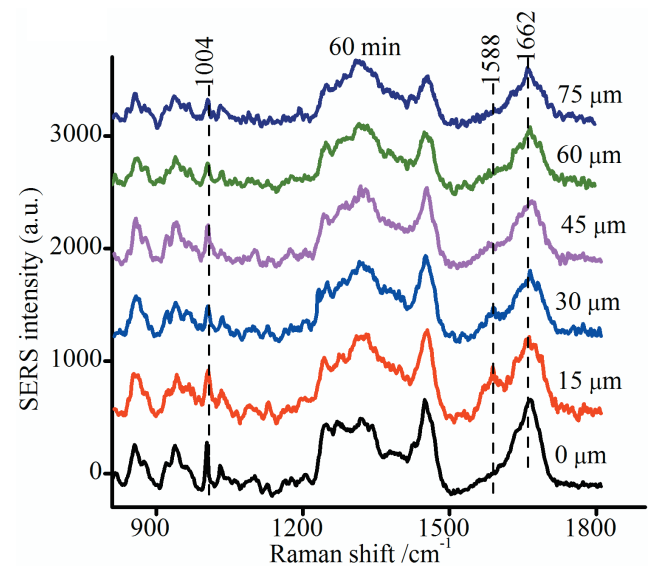

(b)

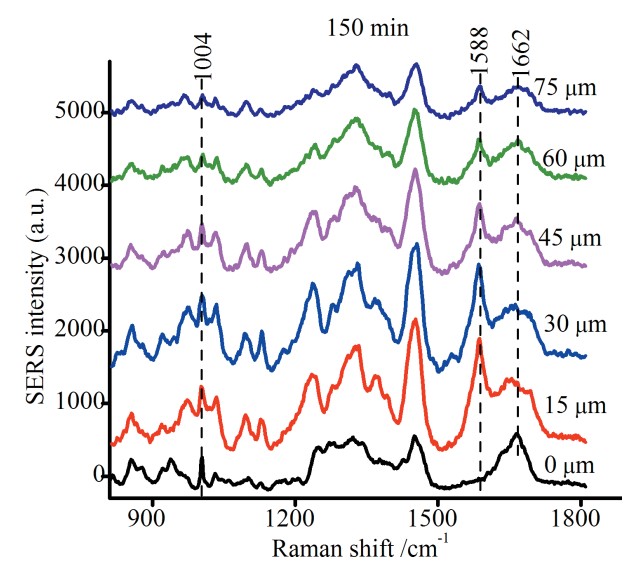

(d)

Fig. 5. A series of SERS spectra of rat skin at $0,15,30,45,60$, and $75 \mu \mathrm{m}$ below the surface after the application of GNPs at various time intervals of (a) 30, (b) 60, (c) 90, and (d) $150 \mathrm{~min}$. The peak at $1588 \mathrm{~cm}^{-1}$ from citrate group was used as GNA marker.

by Eq. (3), the signals can be detected from 15, 30, and $45 \mu \mathrm{m}$ down to 21,42 , and $63 \mu \mathrm{m}$. Given that the epidermis/dermis interface of rat skin used in our experiments was located at the depth between 60 and $80 \mu \mathrm{m}$ (Fig. 2), these data indicated that 20 nm GNPs can penetrate through the epidermis layer, reach the epidermis/dermis interface, and form GNAs after 90 min exposure to GNPs.

After the topical application of $150 \mathrm{~min}$, the citrate group marker at $1588 \mathrm{~cm}^{-1}$ were all detected at 0,15 , 30, 45, 60, and $75 \mu \mathrm{m}$ (measured optical depth: $\Delta$ ) under the skin surface (Figs. 5(c) and 5(d)). After correction by Eq. (3), the signals can be detected from $15,30,45,60$, and $75 \mu \mathrm{m}$ down to $14,28,42,63$, and $105 \mu \mathrm{m}$. These data indicated that $20 \mathrm{~nm}$ GNPs can penetrate through the epidermis layer and into the dermis layer, and form GNAs after 150 min exposure to GNPs.
The results of this study suggested that SERS technology can rapidly and ultra-sensitively monitor the penetration and accumulation of GNPs in the skin. In addition, we observed that penetration depth, penetration time, and SERS enhancements were not relatively correlated, because each GNA has a unique size and shape.

SERS spectroscopy had the potential to monitor the dynamics of nanoparticle penetration and their accumulation within tissues. It was worthy of notice that SERS technology was a high resolution measure method and SERS studies could be carried out in vivo, noninvasively, and in real time. The technique revealed that nanoparticles penetrated into skin, and accumulated in different layers and structures, and $20 \mathrm{~nm}$ GNPs had excellent penetration abilities in skin. The results of our study demonstrated that SERS spectroscopy was an 
objective method for monitoring nanoparticle accumulation in biological tissues. This technology can be a useful tool for evaluating the risks of nanomaterial contamination by cutaneous accidental contact and for assessing therapeutic effect as a vehicle in transdermal drug delivery.

\section{Acknowledgments}

This work was supported by the National Natural Science Foundation of China (No. 61275187, No. 61378089, and No. 31300691).

\section{References}

1. P. G. Marilyn F. Hallock, L. DiBerardinis, D. Kallin, "Potential risks of nanomaterials and how to safely handle materials of uncertain toxicity," J. Chem. Health Saf. 16, 16-23 (2009).

2. H. Arami, A. Khandhar, D. Liggitt, K. M. Krishnan, "In vivo delivery, pharmacokinetics, biodistribution and toxicity of iron oxide nanoparticles," Chem. Soc. Rem. 44, 8576-8607 (2015).

3. N. L. Flaherty, A. Chandrasekaran, M. d. P. S. Pena, G. A. Roth, S. A. Brenner, T. J. Begley, J. A. Melendez, "Comparative analysis of redox and inflammatory properties of pristine nanomaterials and commonly used semiconductor manufacturing nanoabrasives," Toxicol. Lett. 239, 205-215 (2015).

4. R. J. Uzuriaga-Sanchez, S. Khan, A. Wong, G. Picasso, M. Isabel Pividori, M. D. P. Taboada Sotomayor, "Magnetically separable polymer (MagMIP) for selective analysis of biotin in food samples," Food Chem. 190, 460-467 (2016).

5. E. Yan, M. Cao, Y. Wang, X. Hao, S. Pei, J. Gao, Y. Wang, Z. Zhang, D. Zhang, "Gold nanorods contained polyvinyl alcohol/chitosan nanofiber matrix for cell imaging and drug delivery," Mat. Sci. Eng. C: Mater. Biol. Appl. 58, 1090-1097 (2016).

6. M. Faraday, "The Bakerian lecture: Experimental relations of gold (and other metals) to light," Philos. Trans. R. Soc. Lond. B 147, 145-181 (1857).

7. T. W. Prow, J. E. Grice, L. L. Lin, R. Faye, M. Butler, W. Becker, E. M. Wurm, C. Yoong, T. A. Robertson, H. P. Soyer, M. S. Roberts, "Nanoparticles and microparticles for skin drug delivery," Adv. Drug Deliv. Rev. 63, 470-491 (2011).

8. N. A. Monteiro-Riviere, K. Wiench, R. Landsiedel, S. Schulte, A. O. Inman, J. E. Riviere, "Safety evaluation of sunscreen formulations containing titanium dioxide and zinc oxide nanoparticles in UVB sunburned skin: An in vitro and in vivo study," Toxicol. Sci. 123, 264-280 (2011).
9. L. Shi, J. Shan, Y. Ju, P. Aikens, R. K. Prud'homme, "Nanoparticles as delivery vehicles for sunscreen agents," Colloid. Surf. A 396, 122-129 (2012).

10. M. E. Darvin, K. Koenig, M. Kellner-Hoefer, H. G. Breunig, W. Werncke, M. C. Meinke, A. Patzelt, W. Sterry, J. Lademann, "Safety assessment by multiphoton fluorescence/second harmonic generation/ hyper-Rayleigh scattering tomography of $\mathrm{ZnO}$ nanoparticles used in cosmetic products," Skin Pharmacol. Phys. 25, 219-226 (2012).

11. Y. Zhu, C.-S. Choe, S. Ahlberg, M. C. Meinke, U. Alexiev, J. Lademann, M. E. Darvin, "Penetration of silver nanoparticles into porcine skin ex vivo using fluorescence lifetime imaging microscopy, Raman microscopy, and surface-enhanced Raman scattering microscopy," J. Biomed. Opt. 20, 051006 (2015).

12. H. I. Labouta, D. C. Liu, L. L. Lin, M. K. Butler, J. E. Grice, A. P. Raphael, T. Kraus, L. K. El-Khordagui, H. P. Soyer, M. S. Roberts, "Gold nanoparticle penetration and reduced metabolism in human skin by toluene," Pharm. Res. 28, 2931-2944 (2011).

13. F. F. Larese, F. D'Agostin, M. Crosera, G. Adami, N. Renzi, M. Bovenzi, G. Maina, "Human skin penetration of silver nanoparticles through intact and damaged skin," Toxicology 255, 33-37 (2009).

14. B. Baroli, M. G. Ennas, F. Loffredo, M. Isola, R. Pinna, M. A. López-Quintela, "Penetration of metallic nanoparticles in human full-thickness skin," $J$. Invest. Dermatol. 127, 1701-1712 (2007).

15. M. A. Sirotkina, M. V. Shirmanova, M. L. Bugrova, V. V. Elagin, P. A. Agrba, M. Y. Kirillin, V. A. Kamensky, E. V. Zagaynova, "Continuous optical coherence tomography monitoring of nanoparticles accumulation in biological tissues," J. Nanopart. Res. 13, 283-291 (2011).

16. G. Sonavane, K. Tomoda, A. Sano, H. Ohshima, H. Terada, K. Makino, "In vitro permeation of gold nanoparticles through rat skin and rat intestine: Effect of particle size," Colloids Surf. B 65, 1-10 (2008).

17. G. Braun, I. Pavel, A. R. Morrill, D. S. Seferos, G. C. Bazan, N. O. Reich, M. Moskovits, "Chemically patterned microspheres for controlled nanoparticle assembly in the construction of SERS hot spots," $J$. Am. Chem. Soc. 129, 7760-7761 (2007).

18. Y. Lu, G. L. Liu, J. Kim, Y. X. Mejia, L. P. Lee, "Nanophotonic crescent moon structures with sharp edge for ultrasensitive biomolecular detection by local electromagnetic field enhancement effect," Nano Lett. 5, 119-124 (2005).

19. H. X. Xu, J. Aizpurua, M. Käll, P. Apell, "Electromagnetic contributions to single-molecule sensitivity in surface-enhanced Raman scattering," Phys. Rev. E 62, 4318-4324 (2000). 
20. J. P. Camden, J. A. Dieringer, Y. Wang, D. J. Masiello, L. D. Marks, G. C. Schatz, R. P. Van Duyne, "Probing the structure of single-molecule surface-enhanced Raman scattering hot spots," J. Am. Chem. Soc. 130, 12616-12617 (2008).

21. S. M. Stranahan, K. A. Willets, "Super-resolution optical imaging of single-molecule SERS Hot spots," Nano Lett. 10, 3777-3784 (2010).

22. Z. Zhu, T. Zhu, Z. Liu, "Raman scattering enhancement contributed from individual gold nanoparticles and interparticle coupling," Nanotechnology 15, 357 (2004).

23. K. Kneipp, Y. Wang, H. Kneipp, L. T. Perelman, I. Itzkan, R. Dasari, M. S. Feld, "Single molecule detection using surface-enhanced Raman scattering (SERS)," Phys. Rev. Lett. 78, 1667-1670 (1997).

24. K. Kneipp, A. S. Haka, H. Kneipp, K. Badizadegan, N. Yoshizawa, C. Boone, K. E. Shafer-Peltier, J. T. Motz, R. R. Dasari, M. S. Feld, "Surface-enhanced Raman Spectroscopy in single living cells using gold nanoparticles," Appl. Spectrosc. 56, 150-154 (2002).

25. J. Kneipp, H. Kneipp, B. Wittig, K. Kneipp, "Novel optical nanosensors for probing and imaging live cells," Nanomedicine 6, 214-226 (2010).

26. W. R. Premasiri, D. T. Moir, M. S. Klempner, N. Krieger, G. Jones, L. D. Ziegler, "Characterization of the Surface Enhanced Raman Scattering (SERS) of bacteria," J. Phys. Chem. B 109, 312-320 (2005).

27. J. Kneipp, H. Kneipp, M. McLaughlin, D. Brown, K. Kneipp, "In vivo molecular probing of cellular compartments with gold nanoparticles and nanoaggregates," Nano Lett. 6, 2225-2231 (2006).

28. B. Sharma, K. Ma, M. R. Glucksberg, R. P. Van Duyne, "Seeing through bone with surface-enhanced spatially offset Raman spectroscopy," J. Am. Chem. Soc. 135, 17290-17293 (2013).

29. E. C. Dreaden, A. M. Alkilany, X. Huang, C. J. Murphy, M. A. El-Sayed, "The golden age: Gold nanoparticles for biomedicine," Chem. Soc. Rev. 41, 2740-2779 (2012).

30. G. Frens, "Controlled nucleation for the regulation of the particle size in monodisperse gold suspensions," Nature 241, 20-22 (1973).

31. N. J. Everall, "Modeling and measuring the effect of refraction on the depth resolution of confocal Raman microscopy," Appl. Spectrosc. 54, 773-782 (2000).

32. A. Tfayli, O. Piot, M. Manfait, "Confocal Raman microspectroscopy on excised human skin: Uncertainties in depth profiling and mathematical correction applied to dermatological drug permeation," J. Biophotonics 1, 140-153 (2008).

33. A. M. Schwartzberg, C. D. Grant, A. Wolcott, C. E. Talley, T. R. Huser, R. Bogomolni, J. Z. Zhang, "Unique gold nanoparticle aggregates as a highly active surface-enhanced Raman scattering substrate," J. Phys. Chem. B 108, 19191-19197 (2004).

34. S. E. J. Bell, M. R. McCourt, "SERS enhancement by aggregated Au colloids: Effect of particle size," Phys. Chem. Chem. Phys. 11, 7455-7462 (2009).

35. M. Quinten, "Local fields close to the surface of nanoparticles and aggregates of nanoparticles," Appl. Phys. B 73, 245-255 (2001).

36. S. E. Lee, K. J. Choi, G. K. Menon, H. J. Kim, E. H. Choi, S. K. Ahn, S. H. Lee, "Penetration pathways induced by low-frequency sonophoresis with physical and chemical enhancers: Iron Oxide nanoparticles versus lanthanum nitrates," J. Invest. Dermatol. 130, 1063-1072 (2010).

37. M. Kerker, O. Siiman, L. A. Bumm, D. S. Wang, "Surface enhanced Raman scattering (SERS) of citrate ion adsorbed on colloidal silver," Appl. Opt. 19, 3253-3255 (1980).

38. K. Kneipp, H. Kneipp, R. Manoharan, E. B. Hanlon, I. Itzkan, R. R. Dasari, M. S. Feld, "Extremely large enhancement factors in surface-enhanced Raman scattering for molecules on colloidal gold clusters," Appl. Spectrosc. 52, 1493-1497 (1998).

39. S. A. Khan, A. K. Singh, D. Senapati, Z. Fan, P. C. Ray, "Targeted highly sensitive detection of multi-drug resistant salmonella DT104 using gold nanoparticles," Chem. Commun. 47, 9444-9446 (2011).

40. H. Yuan, Y. Liu, A. M. Fales, Y. L. Li, J. Liu, T. VoDinh, "Quantitative surface-enhanced resonant raman scattering multiplexing of biocompatible gold nanostars for in vitro and ex vivo detection," Anal. Chem. 85, 208-212 (2013).

41. J. K. Register, A. M. Fales, H.-N. Wang, S. J. Norton, E. H. Cho, A. Boico, S. Pradhan, J. Kim, T. Schroeder, N. A. Wisniewski, B. Klitzman, V.-D. Tuan, "In vivo detection of SERS-encoded plasmonic nanostars in human skin grafts and live animal models," Anal. Bioanal. Chem. 407, 8215-8224 (2015).

42. M. Moskovits, "Surface-enhanced Raman spectroscopy: A brief retrospective," J. Raman Spectrosc. 36, 485-496 (2005).

43. K. Kneipp, H. Kneipp, J. Kneipp, "Surface-enhanced Raman scattering in local optical fields of silver and gold nanoaggregatess - From single-molecule Raman spectroscopy to ultrasensitive probing in live cells," Acc. Chem. Res. 39, 443-450 (2006).

44. S. E. J. Bell, N. M. S. Sirimuthu, "Surface-enhanced Raman spectroscopy as a probe of competitive binding by anions to citrate-reduced silver colloids," J. Phys. Chem. A 109, 7405-7410 (2005).

45. M. Egawa, Y. Sato, "In vivo evaluation of two forms of urea in the skin by Raman spectroscopy after application of urea-containing cream," Skin Res. Technol. 21, 259-264 (2015). 
46. P. J. Caspers, G. W. Lucassen, R. Wolthuis, H. A. Bruining, G. J. Puppels, "In vitro and in vivo Raman spectroscopy of human skin," Biospectroscopy 4, S31-S39 (1998).
47. D. Huang, W. Zhang, H. Zhong, H. Xiong, X. Guo, Z. Guo, "Optical clearing of porcine skin tissue in vitro studied by Raman microspectroscopy," $J$. Biomed. Opt. 17, 015004 (2012). 